\title{
Radio, Infrared and X-Ray Observations of GRS 1915+105
}

\author{
R. P. Fender \\ Astronomy Centre, University of Sussex, Brighton BN1 9QH U.K. \\ G. G. Pooley \\ MRAO, Cavendish Laboratory, Cambridge CB3 OHE, U.K. \\ C. R. Robinson, B. A. Harmon, S. N. Zhang \\ MSFC, Huntsville, AL 35812, U.S.A. \\ C. Canosa \\ Astronomy Centre, University of Sussex, Brighton BN1 9QH U.K.
}

\begin{abstract}
.
We present multiwavelength observations of the superluminal jet source GRS $1915+105$ in 1996 April-May, over which period a variety of phenomena, including radio QPO, strong infrared emission lines and rapid X-ray flickering and outbursts were observed.
\end{abstract}

\section{Introduction}

GRS $1915+105$ is an energetic X-ray transient with associated relativistic jets (e.g. Mirabel \& Rodriguez 1994). The source undergoes recurrent outbursts with correlated radio - X-ray behaviour (Foster et al 1996). There is no optical counterpart but spectroscopy of a variable infrared counterpart has revealed HI \& HeI emission lines during a period of outburst (Castro-Tirada et al 1996).

GRS $1915+105$ is now being monitored in the radio, soft \& hard X-ray regimes by the Ryle Telescope (RT), XTE/ASM and GRO/BATSE respectively. We combine these data sets for the period 1996 April-May, during which a deep infrared spectrum of the source was also obtained.

\section{Results}

Fig 1 presents the RT, ASM and BATSE monitoring of GRS 1915+105 over the period 1996 April-May, indicating the date on which we obtained our infrared spectrum and when radio QPO were observed. Lack of space precludes a discussion; we only summarise the behaviour of the source in each energy regime. 


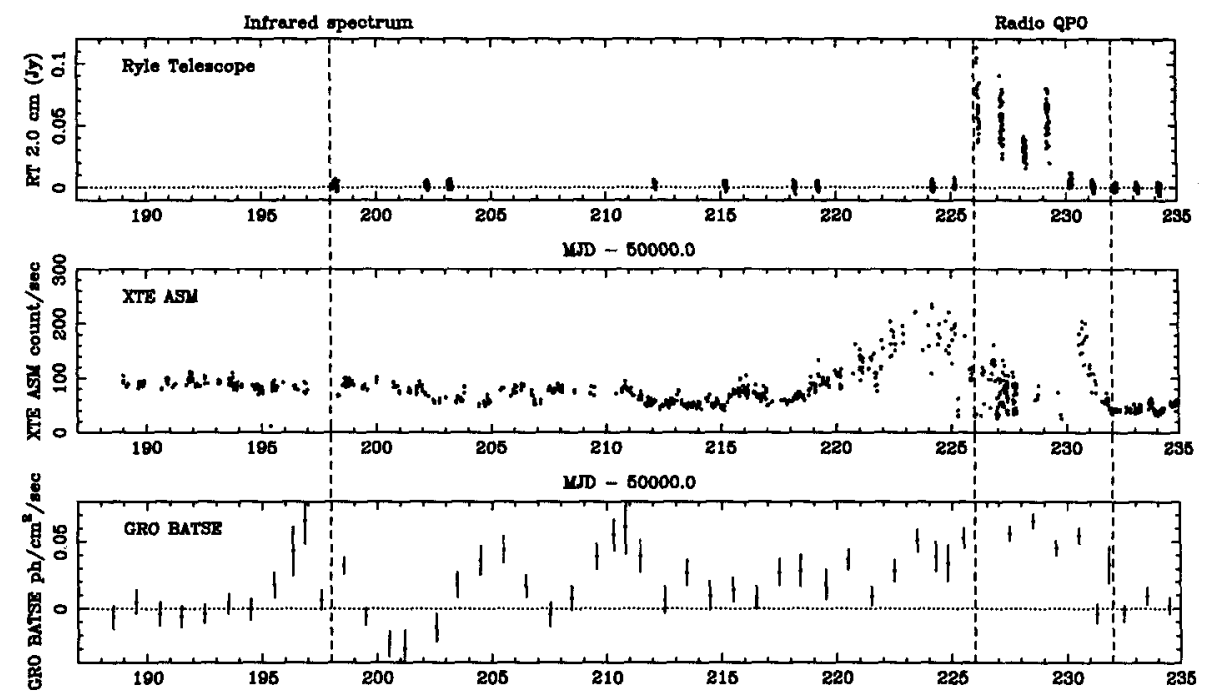

Figure 1. Radio, XTE (ASM) and GRO (BATSE) monitoring of GRS $1915+105$ over the period April-May 1996, indicating when our IR spectrum was obtained and when radio QPO were observed.

- Radio : GRS $1915+105$ remained below detection levels $(\sim 0.5 \mathrm{mJy})$ with the RT until May 23 when it underwent a rapid flare event. Between May 23-27 the source exhibited radio QPO with periods in range $20-100 \mathrm{~min}$.

- Infrared : the UKIRT IR spectrum obtained revealed (at least) strong HeI $2.06 \mu \mathrm{m}$ and HI $2.16 \mu \mathrm{m}$ emission.

- Soft X-ray : GRS 1915+105 had been in a gradual decline until May 20, when it began brightening. During the period of the radio QPO many large amplitude, rapid brightness variations were observed (see also Greiner this proceedings).

- Hard X-ray : the hard X-ray brightness of GRS $1915+105$ varied over the entire 1996 April-May period, but shows activity around the period of the radio flare, with a steady increase in flux up to $\sim 27$ May, when the flux again declined.

Acknowledgments. Thanks to ASM RXTE team for quick-look results.

\section{References}

Castro-Tirada, A. J. et al. 1996, ApJ, 461, L99

Foster R. S. et al. 1996, ApJ, 467, L81

Mirabel, I. F., Rodriguez, L. F. 1994, Nature, 371, 46 